\title{
Fetal Alcohol Spectrum Disorders
}

\section{Commentary}

Alcohol use is now the third leading risk factor for worldwide global disease burden, in terms of disability adjusted life years (Lim et al., 2012). In the developed world, about $40 \%$ of women of childbearing age drink alcohol, and many are drinking at or around the time of conception (Maier \& West, 2001; Paintner, Williams, \& Burd, 2012a). Rates of alcohol use among women are increasing across much of the world. Current prevalence estimates suggest that several hundred thousand individuals with fetal alcohol spectrum disorders (FASD) are born each year (May, Gossage, \& Kalberg, 2009). This is especially tragic in light of the fact that we have effective prevention methods as well as office-based interventions for prenatal alcohol exposure.

A look back enhances our view of the future. In 1899, William Sullivan, a medical officer at the Convict Prison in England, reported on the contribution of maternal alcohol use to infant mortality (Sullivan, 1899). He reported that of the 600 children born to 100 incarcerated women, 55.8\% were born dead or died before the age of two. Mortality rates for later-born children were double what they were for first-born children. He also demonstrated a beneficial effect on mortality risk for women who quit drinking during pregnancy. Around the turn of the century, a sophisticated public education program was implemented by the Anti-Saloon League in the United States (Sanders et al., 2009; Anti-Saloon League, 1909). The focus of the Anti-Saloon league was on the impact of alcohol use on the family and society. Interestingly, they also emphasized the role of prenatal exposure as a risk marker for increased mortality rates for infants and children, for growth impairments, and for learning and developmental deficits. They had a prescient anticipation of two of the current diagnostic criteria for FASD (growth impairment, neurocognitive deficits) (Stratton et al., 1996).

Clearly, there is broad overlap between those earlier findings and the data being published over 100 years later. Both epochs of research on prenatal alcohol exposure emphasize the risk of drinking prior to and during pregnancy for maternal, infant and child health, and the tragic impact of alcohol abuse on family functioning, birth defects, growth impairments and neurocognitive deficits. Public policy has also completed the circle, as evidenced by a 1931 monograph on prenatal care from the Children's Bureau of the U. S. Department of Labor, which recommended that during pregnancy, "alcoholic drinks should be avoided entirely” (U.S. Dept of Labor, 1931).

\section{A Conceptual Model of Neurocognitive Impairments - Alcohol Related Neurodevelop- mental Disorder}

Neurocognitive impairments represent a unifying symptom criterion across three of the four fetal alcohol spectrum disorders categories: fetal alcohol syndrome; partial fetal alcohol syndrome; and alcohol-related neurodevelopmental disorder (ARND). The most prevalent FASD diagnostic category is ARND, with a prevalence rate of five to seven times the rate of fetal alcohol syndrome (NIAAA, 2011; Sampson et al., 1997). This proportion supports the idea that ARND deserves increased attention in future discussions of FASD. What does application of the term ARND imply in a clinical setting? What clinical need is it meant to fill that is not filled by FAS? Is it any more specific than the encompassing term FASD? While the diagnosis of ARND is complex due to the highly variable phenotypic presentation, progress is being made in developing improved diagnostic methods for ARND (NIAAA, 2011; Sampson et al., 1997).

The label "alcohol-related neurodevelopmental disorder" suggests the following:

- Alcohol: Prenatal exposure is necessary for the diagnosis.

- Related: Implies association, which is inclusive of causation; alcohol may be a key link in a multielement causal chain.

- Neuro: Indicates dysfunction of or damage to the CNS, leading to, or transacting with, developmental impairments.

- Developmental: The condition is genetically programmed, interacting over time with social and physical environmental influences and determinants, within limits of expression that are defined by genetic canalization.

- Mental: The mental in developmental. This is a diagnosis pertaining to the cognitive, emotional, social, and conative emergents of brain function.

- Disorder: A departure from the average, idealized, normative, expectable, or predictable order of developmental function, taking into account the concept of dynamic change over time. 
Prenatal alcohol exposure (PAE) can be conceptualized as a teratogen acting upon the genome and epigenome, and lowering the expression threshold for neurological disorders, mental disorders, developmental delays, and structural abnormalities of the central nervous system (Paintner et al., 2012b). In addition, PAE may act as an effect modifier by increasing risk for, and severity of, adverse outcomes from a wide range of genetic and environmental exposures. These mechanisms also increase rates of comorbidity for a broad range of neuropsychiatric outcomes, contributing to the highly variable endophenotype associated with PAE (Burd et al., 2003a). Many of the findings that define the ARND phenotype are recognized developmental disorders and mental disorders (NIAAA, 2011; Burd, 2003b; Burd et al., 2007). The recent revision of the Diagnostic and Statistical Manual of Mental Disorders which adds the category neurobehavioral disorder associated with prenatal alcohol exposure represents an important step in improving the diagnostic capacity for FASD (American Psychiatric Association, 2013). Phenotype assessment is further complicated by the nearly ubiquitous presence of additional prenatal risk factors, including maternal smoking, nutrition, genetics, other epigenetic factors, drug abuse, maternal age, increased parity, poverty, and parental mental health disorders. These factors often confound detection and quantification of the effects of PAE.

\section{FASD and ARND: Age and Development Dependent Increases in Phenotype Complexity and Severity}

The complex multisystem interactions in ARND and its more encompassing predecessor, FASD, are both age and development dependent, and both of these variables are crucial in the assessment of these impairments (Burd et al., 2003a). A useful example is the complexity of assessment of communication function in infants and in persons with severe developmental delays. Assessment of psychosis and depression is also complex in these populations. Thus, we are faced with the problem of how to diagnose the adverse outcomes from PAE in tens of thousands of children and adolescents who may have neurobehavioral phenotypes that are etiologically related to PAE, which are influenced as well by multiple other environmental and genetic factors including, frequently, poly-substance abuse.

The window of time during which effects from these influences emerge begins in infancy and extends through adolescence (Paintner et al., 2012a; Streissguth et al., 1996). The etiological complexity of effect is often further complicated by postnatal exposure to multiple effect modifiers of the individual's phenotype. Among the most important of these are the effects of early altered developmental trajectories, which impact the course of later development in the form of delay and/or deviation, especially in cognitive and social developmental lines. This mix of factors results in a need for lengthy neurodevelopmental therapies, which have both positive and negative influences on outcome. The potential for negative impacts from multiple interventions during development has not been adequately explored.
Discrepancies among an individual's chronological age, developmental level, and age-determined social expectations tend to magnify the number and severity of his or her comorbidities within the realms of cognitive and social abilities. In short, PAE is not only a determining, but also an interacting and transacting element in a complex multifactorial causal chain of effect modifiers, which are difficult to partition out in clinical settings.

\section{Secondary Disabilities}

The effects described above continue to impact development into adolescence and adulthood. Streisguth et al. (1996) have conceptualized the interplay of these postnatal developmental adversities and impaired outcomes as secondary disabilities. As development proceeds, these often take center stage as a key component of phenotype severity. Secondary disabilities are potentially preventable, if assessment and treatment planning for affected people is oriented towards their future.

\section{FASD, ARND and Foster Care}

Few service systems have as close an involvement with substance abuse and its consequences as the foster care system. The overwhelming majority of children in foster care are there because of parental substance abuse (Burd et al., 2011). Every child entering foster care needs to be screened for FASD, including ARND (Burd et al., 2011). Access to diagnostic services is urgently needed in this system of care, as is improved training to help staff serve children and families affected by FASD and especially ARND. One model effort has been underway for nearly a decade by the infant-health nonprofit Zero to Three, working in cooperation with local court teams in several states (Burd et al., 2011). This program focuses on changing how young children and their families enter into the foster care system. The outcome data suggest that this model should be widely emulated (McCombs-Thornton \& Foster, 2012).

\section{FASD, ARND and Mental Illness}

FASD appears to be a uniquely important risk marker for expression, severity and comorbidity of mental disorders across the lifespan (NIAAA, 2011; Burd, 2007; O’Connor, 2009). However, awareness of FASD and access to relevant diagnostic services in the mental health community is limited. It is urgent that the diagnostic criteria for FASD, especially its subset, ARND, be modified to meet the needs of office-based practices in the mental health services community. A recent consensus conference on ARND has begun this effort (NIAAA, 2011). It must continue to solicit input from neurologists, psychiatrists, psychologists, and other mental health providers who have extensive clinical contact with people with FASD and especially ARND, as it is crucial that the refined criteria meet the needs of these providers and have an increased emphasis on ARND. There are simply too many people with ARND born each year for the current multidisciplinary model to serve the majority of them. 


\section{FASD, ARND and Corrections Systems}

"We can envision few things more certainly beyond one's control than the drinking habits of a parent prior to one's birth" Dilbeck $v$. State 643 So $2 d 1027$ Fla 1994 (1994)

FASD is a condition of special significance to the corrections system. Adolescents with FASD are 19 times more likely to enter the corrections system than unaffected adolescents (Popova, 2011). The confluence of substance abuse, impaired decision making, and multiple other social adversities predispose them to contact with the legal system (Burd et al., 2010). Huge efforts are needed to improve screening and access to diagnostic services for FASD in these systems, where a vanishingly small number of affected people are identified (Burd et al., 2004a). Identification of FASD, with increased attention to ARND, has important implications at every step of the legal system. Appropriate diagnosis and treatment have the potential to enhance diversion efforts, decrease rates of incarceration, improve the corrections experience, improve parole and probation outcomes, and decrease recidivism. In no area of society is increased emphasis on ARND/FASD more needed (Burd et al., 2010). The journal Psychiatry and Law has begun this effort with two recent special issues on FASD, but a greater emphasis, originating from leadership within the legal and corrections systems, is needed.

\section{Substance Abuse Treatment and ARND/ FASD}

FASD is an important marker for substance abuse developing in childhood and adolescence and continuing into adult life (Streissguth, 2004). Current substance abuse treatment programs need to be modified to enhance screening for ARND/FASD and to implement approaches which emphasize matching intervention strategies, to accommodate the neuropsychiatric impairments of people with ARND. This strategy has the potential to improve treatment outcomes for people entering substance abuse treatment who have ARND, nearly all of whom go unrecognized. Treatment failure has serious health, vocational and often legal consequences for parents and their children. For parents, these may include permanent loss of custody of their children. Treatment failures in women increase risk of recurrent exposure for subsequent pregnancies. This, in turn, increases the recurrence risk for ARND/FASD in younger siblings and the risk of fetal, infant and child mortality (Burd et al., 2004b). For the mothers, having a child with FASD is also an important marker for increased mortality risk (Li et al., 2011). Model programs with demonstrated efficacy are available and are currently being implemented in multiple locations (Grant et al., 2005).

\section{Policy Development in ARND/FASD}

Cost and prevalence studies are essential for determining governmental policy priorities, amid the inevitable competition for resource allocation. Positive developments are occurring on these fronts. Prevalence data is proliferating, and estimates are now available from many countries across the world; see May et al. (2009) for a review. As well, a new generation of comprehensive cost studies for FASD is underway (Popova et al., 2012). This information will be crucial to support improved decision making by the range of governmental agencies whose portfolios include FAS and other FASD.

\section{Prevention Strategies}

Prevention efforts should focus on multiple domains. In North Dakota, we have focused on improving ascertainment of prenatal alcohol use in prenatal care settings. We screen with a single question- "When was your last drink?"-which we have found to have epidemiologic performance criteria equal to, or exceeding, other commonly used prenatal screening tools. We have also focused on prevention of recurrent cases born to the same mother. Our current data suggest a decrease of FASD of between $20 \%$ and $30 \%$ over the past five years. This will result in huge savings - both financial and in terms of misery prevented-across the lifespan of the unaffected people.

\section{Conclusions}

In this issue of the Journal, the authors report on recent and important research findings on FASD. As we enter our second century of research on prevention of PAE and FASD, our progress and outcomes need to be scaled to prevent hundreds of thousands of new cases (most of which will involve an ARND) and improve the lives of the several million affected people and their families. This is a worldclass agenda, with worldwide application and worldwide consequences.

\section{References}

American Psychiatric Association. (2013). Diagnostic and statistical manual of mental disorders (5th ed.). Arlington, VA: American Psychiatric Publishing.

Anti-Saloon League (1909). Retrieved from http://www. wlp.lib.oh.us/AntiSaloon/index.html.

Burd, L., Carlson, C., Kerbeshian, J. (2007). Fetal Alcohol Spectrum Disorders and Mental Illness. International Journal on Disability and Human Development. 6, 383-396.

Burd, L., Cohen, C., Shah, R., Norris, J. (2011, Spring). A court team model for care of young children in foster care: The role of prenatal alcohol exposure and fetal alcohol spectrum disorders. The Journal of Psychiatry and Law, 39, 179-191.

Burd, L., Cotsonas-Hassler, T. M., Martsolf, J. T., Kerbeshian, J. (2003b). Recognition and management of fetal alcohol syndrome. Neurotoxicology and Teratology, 25, 681-688.

Burd, L., Fast, D. K., Conry, J, Williams, A. (2010, Winter). Fetal Alcohol Spectrum Disorder as a marker for increased risk of involvement with correction systems. The Journal of Psychiatry and Law, 38, 559583.

Burd, L., Klug, M. G., Martsolf, J. T., Kerbeshian, J. (2003a). Fetal Alcohol Syndrome: Neuropsychiatric 
phenomics. Neurotoxicology and Teratology, 25, 697705.

Burd, L., Selfridge, R. H., Klug, M. G., Bakko, S. A. (2004a). Fetal Alcohol Syndrome in the United States corrections system. Addiction Biology, 9, 169-176.

Burd, L., Wilson, H. (2004b). Fetal, infant, and child mortality in a context of alcohol use. American Journal of Medical Genetics Part C: Seminars in Medical Genetics, 127C, 51-58.

Grant, T., Ernst, C., Streissguth, A., Stark, K. (2005). Preventing alcohol and drug exposed births in Washington State: Intervention findings from three Parent-Child Assistance Program sites. The American Journal of Drug and Alcohol Abuse, 31, 471-490.

Li, Q., Fisher, W. W., Peng, C. Z., Williams, A.D., \& Burd, L. (2011). Fetal alcohol spectrum disorders: A population-based study of premature mortality rates in the mothers. Maternal and Child Health Journal doi: 10.1007/s10995-011-0844-3.

Lim, S. S., Vos, T., Flaxman, A. D., Danaei, G., Shibuya, K., Adair-Rohani, H., . . Ezzati, M. (2012, December 15). A comparative risk assessment of burden of disease and injury attributable to 67 risk factors and risk factor clusters in 21 regions, 1990-2010: A systematic analysis for the Global Burden of Disease Study 2010. The Lancet, 380, 2224-2260 doi: 10.1016/S0140-6736(12)61766-9.

Maier, S. E., West, J. R. (2001). Drinking patterns and alcohol-related birth defects. Alcohol Research and Health, 25, 168-174.

May, P. A., Gossage, J. P., Kalberg, W. O., et al. (2009). Prevalence and epidemiologic characteristics of FASD from various research methods with an emphasis on recent in-school studies. Developmental Disabilities Research Reviews, 15, 176-192.

McCombs-Thornton, K. L., Foster, E. M. (2012). The effect of the ZERO TO THREE Court Teams initiative on types of exits from the foster care system-A competing risks analysis. Children and Youth Services Review, 34, 169-178. doi:10.1016/j.childyouth.2011. 09.013.

National Institute on Alcohol Abuse and Alcoholism (2011). Consensus Statement: Recognizing alcoholrelated neurodevelopmental disorder (ARND) in primary health care of children. Retrieved from http://www.niaaa.nih.gov/sites/default/files/ARNDCo nferenceConsensusStatementBooklet_Complete.pdf

O’Connor, M. J., Paley, B. (2009). Psychiatric conditions associated with prenatal alcohol exposure. Developmental Disabilities Research Reviews, 15, 225-234.

Paintner, A., Williams, A. D., Burd, L. (2012a). Fetal alcohol spectrum disorders-Implications for child neurology, Part 1: Prenatal exposure and dosimetry. Journal of Child Neurology, 27, 258-263.

Paintner, A., Williams, A. D., Burd, L. (2012b). Fetal alcohol spectrum disorders-Implications for child neurology, Part 2: Diagnosis and management. Journal of Child Neurology 27, 355-362.

Popova, S., Lange, S., Bekmuradov, D., Mihic, A., Rehm, J. (2011). Fetal alcohol spectrum disorder prevalence estimates in correctional systems: A systematic literature review. Canadian Journal of Public Health, 102, 336-340.

Popova, S., Lange, S., Burd, L., Rehm, J. (2012). Health care burden and cost associated with fetal alcohol syndrome: Based on official Canadian data. PLOS ONE, 7, e43024.

Sampson, P. D., Streissguth, A. P., Bookstein, F. L., Little, R. E., Clarren, S. K., Dehaene, P., Hanson, J. W., \& Graham, J. M. Jr. (1997). Incidence of fetal alcohol syndrome and prevalence of alcohol-related neurodevelopmental disorder. Teratology, 56, 317326.

Sanders, J. L. (2009). Were our forebears aware of prenatal alcohol exposure and its effects? A review of the history of fetal alcohol spectrum disorder. Canadian Journal of Clinical Pharmacology, 16, 288-295.

Stratton, K. R., Howe, C. J., Battaglia, F. C., Institute of Medicine (1996). Fetal Alcohol SyndromeDiagnosis, Epidemiology, Prevention, and Treatment. Washington, D.C., United States: National Academy Press.

Streissguth, A., Barr, H., Kogan, J., Bookstein, F. (1996). Understanding the occurrence of secondary disabilities in clients with fetal alcohol syndrome (FAS) and fetal alcohol effects (FAE). Final report to the Centers for Disease Control and Prevention (CDC). Seattle, WA, United States: University of Washington, Fetal Alcohol and Drug Unit, Tech. Rep. No. 96-06.

Streissguth, A. P., Bookstein, F. L., Barr, H. M., Sampson, P. D., O’Malley, K., Young, J. K. (2004). Risk factors for adverse life outcomes in fetal alcohol syndrome and fetal alcohol effects. Journal of Developmental and Behavioral Pediatrics, 25, 228-238.

Sullivan, W. C. (1899). A note on the influence of maternal inebriety on the offspring. Journal of Mental Science, 45, 489-507.

U.S. Department of Labor Children's Bureau (1931). Prenatal Care: Bureau Publication No. 4. United States Government Printing Office. Washington, D.C.

Larry Burd, PhD

North Dakota Fetal Alcohol Syndrome Center

Department of Pediatrics

University of North Dakota School of Medicine and Health Sciences

Jacob Kerbeshian, MD

Clinical Professor Neuroscience

University of North Dakota School of Medicine and Health Sciences 